\title{
APPLICATIONS OF LAPLACE TRANSFORMS AND THEIR INVERSES
}

\author{
CHARLES FOX ${ }^{1}$
}

\begin{abstract}
By means of Laplace transforms and their inverses we first solve the Varma transform, considered as an integral equation for an unknown function in the integrand. We then express two operators of fractional integration in terms of Laplace and inverse Laplace transforms.
\end{abstract}

1. Introduction. This paper continues from my previous paper [2]. In [8] Varma gives a generalization of the Laplace transform, namely

$$
g(t)=\int_{0}^{\infty}\left[(t u)^{\mu-1 / 2} e^{-(1 / 2) t u} W_{k, \mu}(t u)\right] f(u) d u \quad(t>0),
$$

where $W_{k, \mu}(z)$, Whittaker's function [10, p. 334], may be defined by

$$
W_{k, \mu}(z)=\frac{e^{-(1 / 2) z} z^{k}}{\Gamma\left(\frac{1}{2}-k+\mu\right)} \int_{0}^{\infty} u^{-k-1 / 2+\mu}\left(1+u z^{-1}\right)^{k-1 / 2 \cdot \mu} e^{-u} d u .
$$

It can be shown that $W_{k, \mu}(z)$ exists for all values of $k$ and $\mu$ and all values of $z$ except real negative ones. Special cases of $W_{k, \mu}^{\prime}(z)$ occur in many problems of mathematical physics. When $k+\mu=\frac{1}{2}$, (1) reduces to the Laplace transform of $f(u)$.

(1) becomes an integral equation if $g(t)$ is given and $f(u)$ is to be found. This has been solved by Saxena [4] using the fractional integration operator $\mathcal{R}\{\alpha, \beta: f(x)\}$, defined later in equation (31). Since comprehensive tables of Laplace transforms exist, while tables of $\mathcal{R}\{\alpha, \beta: f(x)\}$ are comparatively rare, I give here a solution of (1), considered as an integral equation for $f(u)$, by means of Laplace transforms and their inverses. After this I show how to express two operators of fractional integration in terms of Laplace operators. The methods used are those used in my previous paper [2].

Received by the editors December 6, 1971.

AMS 1969 subject classifications. Primary 4790, 4420.

Key words and phrases. Laplace transforms, Mellin transforms. eliminatıng ganma function factors from integrands.

${ }^{1}$ This work was supported by the National Research Council of Canada grant 240187. 
2. The Laplace and Mellin transforms. The Laplace transform of $\phi(x)$ is denoted by $\mathfrak{Q}\{\phi(x)\}$ and defined by

$$
\mathscr{L}\{\phi(x)\}=\int_{0}^{\infty} e^{-x t} \phi(x) d x=\psi(t) .
$$

With $\phi(x)$ and $\psi(t)$ related as in (3) the inverse Laplace transform of $\psi(t)$ is written

$$
\mathfrak{L}^{-1}\{\psi(t)\}=\phi(x) .
$$

We assume that $\mathfrak{L}^{-1}$ is found by reading a table of Laplace transforms in reverse. Symbolically we then have

$$
\mathfrak{L}^{-1} \mathfrak{L}=\mathfrak{L}^{-1}=1 .
$$

Usually $\mathfrak{L}$ will change the variable $x$ to $t$ and $\mathfrak{L}^{-1}$ will change $t$ to $x$.

The Mellin transform of $f(u)$ is denoted either by $\mathfrak{M}\{f(u)\}$ or by $F(s)$, whichever is more convenient. Usually $u$ is real and positive while $s$ is a complex variable of the form $s=\sigma+i \tau, \sigma$ and $\tau$ both real. The definition of $\mathfrak{M}\{f(u)\}=F(s)$ is given by

$$
\mathfrak{M}\{f(u)\}=F(s)=\int_{0}^{\infty} f(u) u^{s-1} d u .
$$

Considering $(t u)^{\alpha} f(t u)$ to be a function of $u$, with $t>0$, from (6) we have

$$
\mathfrak{M}\left\{(t u)^{\alpha} f(t u)\right\}=F(s+\alpha) t^{-s} .
$$

If $f(u)$ and $F(s)$ are related as in (6) then the inverse Mellin transform of $F(s)$ can be denoted by $\mathfrak{M}^{-1}\{F(s)\}=f(u)$ or by the equation

$$
f(u)=\frac{1}{2 \pi i} \int_{C^{\prime}} F(s) u^{-s} d s,
$$

where $C$ is a suitable contour in the complex $s$ plane.

If $\mathfrak{M}\{h(u)\}=H(s)$ and $\mathfrak{M}\{f(u)\}=F(s)$ then the Mellin-Parseval theorem states that

$$
\int_{0}^{x} h(u) f(u) d u=\frac{1}{2 \pi i} \int_{C} H(s) F(1-s) d s
$$

where $C$ is a suitable contour in the $s$ plane.

Proofs of (6), (8) and (9) are given in [7, pp. 46, 60 and 94].

With $s=\sigma+i \tau, \sigma$ and $\tau$ both real, if $\sigma>|\mu|-\frac{1}{2}$ then from [5, p. 337, (8)] with $\alpha=\frac{1}{2}$ and $\beta=1$ we have

$$
\mathfrak{M}\left\{e^{-" ~ "} W_{k, \mu}(u)\right\}=\frac{\Gamma\left(-\mu+s+\frac{1}{2}\right) \Gamma\left(\mu+s+\frac{1}{2}\right)}{\Gamma(s-k+1)} .
$$


From (7) we then deduce, if $\sigma>0$ and $\sigma>-2 \mu$, that

$$
\mathfrak{M}\left\{(t u)^{\mu-1 / 2} e^{-(1 / 2) t u} W_{k, \mu}(t u)\right\}=\frac{\Gamma(s) \Gamma(s+2 \mu)}{\Gamma\left(s+\mu-k+\frac{1}{2}\right)} t^{-j},
$$

where we consider that $\mathfrak{M}$ acts upon a function of $u$.

\section{The solution of (1) as an integral equation for $f(u)$.}

THEOREM 1. If (i) $\mu>0, \mu-k>-1$, (ii) $f(x) \in L_{2}(0, \infty)$, (iii) $s^{\mu+k-1 / 2} F(1-s) \in L\left(\frac{1}{2}-i \infty, \frac{1}{2}+i \infty\right)$, (iv) $F(1-s) \in L\left(\frac{1}{2}-i \infty, \frac{1}{2}+i \infty\right)$ and (v) $y^{-1 / 2} f(y) \in L(0, \infty)$ where $f(y)$ is of bounded variation near the point $y=x$, then the solution of (1), as an integral equation for $f(u)$ is

$$
f(x)=x^{-2 \mu} \mathfrak{Q}^{-1}\left\{t^{-\mu-k+1 / 2} \mathfrak{Q}\left[x^{\mu-k+1 / 2} \mathfrak{Q}^{-1}\{g(t)\}\right]\right\} .
$$

Proof. We first apply (9) to the right-hand side of (1). For large positive $u$ and $t>0$ the asymptotic expansion of $W_{k, \mu}(z)[10$, p. 336] gives us

$$
(t u)^{\mu-1 / 2} e^{-(1 / 2) t u} W_{k, \mu}(t u)=(t u)^{\mu+k-1 / 2} e^{-t u}\left\{1+O\left(u^{-1}\right)\right\} .
$$

For small positive $u$ we use the formula for $W_{k, \mu}(u)$ in terms of the $M_{k, \mu}(u)$ and $M_{k,-\mu}(u)$ series given in [10, p. 340], where the two $M$ series are defined in $[\mathbf{1 0}$, p. 332]. We then have for $t>0, u$ positive and small

$$
(t u)^{\mu-1 / 2} e^{--(1 / 2) t u} W_{k, \mu}(t u)=(t u)^{2 \mu}\{1+O(u)\} .
$$

Since, condition (i), $\mu>0$, it follows from (13) and (14) that the expression inside the square braces in (1) belongs to $L_{2}(0, \infty)$, when considered as a function of $u$. This result, combined with condition (ii), $f(u) \in L_{2}(0, \infty)$, justifies our use of Theorem 72, p. 95 of [7], with $k=\frac{1}{2}$, so that we may apply (9) to the right-hand side of (1). Using (11) we have

$$
g(t)=\frac{1}{2 \pi i} \int_{1 / 2-i \infty}^{1 / 2+i \infty} \frac{\Gamma(s) \Gamma(s+2 \mu)}{\Gamma\left(s+\mu-k+\frac{1}{2}\right)} t^{-s} F(1-s) d s \quad(t>0),
$$

where the contour in the $s$ plane is the straight line $s={ }_{2}^{1}+i \tau$ and $\tau$ varies from $-\infty$ to $\infty$.

In the next part of the proof we eliminate the three gamma functions from the integrand of (15) by the methods of my previous paper [2]. From the asymptotic expansion of the gamma function [10, p. 273] along the line $s=\frac{1}{2}+i \tau$, for large $|\tau|$ we have

$$
\frac{\Gamma(s+2 \mu)}{\Gamma\left(s+\mu-k+\frac{1}{2}\right)} F(1-s)=s^{\mu+k-1 / 2} F(1-s)\left\{1+O\left(s^{-1}\right)\right\} .
$$


From condition (iii) the cofactor of $\Gamma(s)$ in the integrand of (15) therefore belongs to $L\left(\frac{1}{2}-\infty, \frac{1}{2}+\infty\right)$. Hence we may use equation (4), p. 300 of [2], with $\alpha=1$ and $\beta=0$, to eliminate $\Gamma(s)$ from the integrand of (15) by means of the operator $\mathfrak{I}^{-1}$. The result is

$$
\mathfrak{L}^{-1}\{g(t)\}=\frac{1}{2 \pi i} \int_{1 / 2-i \propto}^{1 / 2+i \propto} \frac{\Gamma(s+2 \mu)}{\Gamma\left(s+\mu-k+\frac{1}{2}\right)} x^{s-1} F(1-s) d s .
$$

Let $\lambda=\mu-k+\frac{1}{2}$ then from (17) we have

$$
\begin{aligned}
& \mathfrak{L}\left[x^{\lambda} \mathfrak{Q}^{-1}\{g(t)\}\right] \\
& =\int_{0}^{\infty} e^{-t x} x^{\lambda}\left\{\frac{1}{2 \pi i} \int_{1 / 2-i \infty}^{1 / 2+i \infty} \frac{\Gamma(s+2 \mu)}{\Gamma(s+\lambda)} x^{s-1} F(1-s) d s\right\} d x .
\end{aligned}
$$

Since $s=\frac{1}{2}+i \tau$, the real part of the power of $x$ in (18) is $\lambda-\frac{1}{2}=\mu-k$ which, by condition (1), exceeds - 1 . Also, by (16) and condition (iii), the terms in $s$ in (18) belong to $L\left(\frac{1}{2}-i \infty, \frac{1}{2}+i \infty\right)$. Hence, the integral in (18) is an absolutely convergent double integral and we may integrate first with respect to $x$. This eliminates $\Gamma(s+\lambda)$ in the denominator to give us

$$
\mathcal{L}\left[x^{\mu-k+12 \mathfrak{L}^{-1}}\{g(t)\}\right]=\frac{1}{2 \pi i} \int_{1 / 2-i \infty}^{1 / 2+i \infty} \Gamma(s+2 \mu) t^{-s-\mu+k-1 / 2} F(1-s) d s .
$$

Again, conditions (i) and (iv) allow us to use equation (4) of [2], with $\alpha=1$ and $\beta=2 \mu$, and so we apply $\mathfrak{L}^{-1}$ to (19) to eliminate $\Gamma(s+2 \mu)$. The result is

$$
\mathfrak{I}^{-1}\left\{t^{-\mu-k+1 / 2} \mathfrak{Y}\left[x^{\mu-k+1 / 2} \mathfrak{Z}^{-1}\{g(t)\}\right]\right\}=\frac{1}{2 \pi i} \int_{1 / 2-i \infty}^{1 / 2+i \propto} x^{s+2 \mu-1} F(1-s) d s .
$$

This ends the second part of the proof and in the third part we show that the integral in (20) is equal to $x^{2 \mu} f(x)$.

On replacing $s$ by $1-s$ in (20) we have

$$
\begin{aligned}
\frac{1}{2 \pi i} \int_{1 / 2-\imath x}^{1 / 2+i x} x^{\alpha+2 \mu-1} F(1-s) d s & =\frac{1}{2 \pi i} x^{2 \mu} \int_{1 / 2-i \infty}^{1 / 2+i \infty} F(s) x^{-s} d s, \\
& =x^{2 \mu} f(x),
\end{aligned}
$$

since condition (v) allows us to use Theorem 28 , p. 46 of [7] with $k=\frac{1}{2}$. On adding (20) and (22) and dividing by $x^{2 \mu}$ we arrive at (12) and so complete the proof of Theorem 1.

4. Test of (12) as the solution of (1). A simple test is to write $\mu+k=\frac{1}{2}$. (1) then reduces to $g(t)=\mathfrak{L}\{f(x)\}$ and, since $\mathfrak{L}^{-1} \mathfrak{Z}=1$ by equation (5), (12) reduces to $f(x)=\mathbb{L}^{\prime-1}\{g(t)\}$. These results are obviously equivalent to each other. 
For another test let $\mu$ be as in (1) and let

$$
g(t)=2 t^{\mu} K_{2 \mu}\left(2 t^{1 / 2}\right),
$$

where $K_{v}(z)$ is a Bessel function of the second kind [9, p. 78]. From [5, p. 283, (40)], with $\alpha=1$, we have

$$
\mathfrak{L}^{-1}\{g(t)\}=x^{-2 \mu-1} e^{-1 / x} .
$$

From [5, p. 146, (29)], with $\alpha=4$, we then have

$$
\mathfrak{L}\left[x^{\mu-k+1 / 2} \mathfrak{Q}^{-1}\{g(t)\}\right]=2 t^{(1 / 2)(\mu+k-1 / 2)} K_{-\mu-k+1 / 2}\left(2 t^{12}\right) .
$$

From [5, p. 283, (40)], with $\alpha=1$, we then have

$$
x^{-2 \mu} \mathfrak{Q}^{-1}\left\{t^{-\mu-k+1 / 2} \mathfrak{Q}\left[x^{\mu-k+1 / 2} \mathfrak{L}^{-1}\{g(t)\}\right]\right\}=x^{-\mu / k-1 / \cdots 1} e^{-1 x} .
$$

(26) is then the solution of (1) when $g(t)$ is given by (23). Since there are no tables of Varma transforms we cannot check this result directly. But if instead of using (1) we use the equivalent equation (15) a check for (23) can be obtained as follows.

$$
\mathfrak{M}\left\{x^{-\mu+k-1 / 2-1} e^{-1 / x}\right\}=F(s)=\Gamma\left(-s+\mu-k+{ }_{2}^{3}\right) .
$$

On inserting $F(1-s)$ in (15) the gamma function in the denominator is cancelled out. From [5, p. 331, (26)], using manipulations similar to those used in deducing (7) from (6), we find that the resultant integral is equal to $2 t^{\mu} K_{2 \mu}\left(2 t^{1 / 2}\right)$ agreeing with (23). This acts as a check for (26).

5. Fractional integration expressed by means of $\mathfrak{Z}$ and $\mathbb{L}^{1}$ operators. The $\mathfrak{I}$ and $\mathfrak{R}$ types of fractional integration considered here, when acting upon $f(x)$, are defined by

$$
\begin{aligned}
& \mathfrak{J}\{\alpha, \beta: f(x)\}=\frac{x^{-\beta-\alpha}}{\Gamma(\alpha)} \int_{0}^{x}(x-u)^{x-1} u^{\beta} f(u) d u, \\
& \mathcal{R}\{\alpha, \beta: f(x)\}=\frac{x^{\beta}}{\Gamma(\alpha)} \int_{x}^{\infty}(u-x)^{\alpha-1} u^{-\beta-\alpha} f(u) d u .
\end{aligned}
$$

Most other types of fractional integration can be derived from these two.

The Mellin transforms are related as follows:

$$
\begin{aligned}
& \mathfrak{M}[\Im\{\alpha, \beta: f(x)\}]=\frac{\Gamma(\beta+1-s)}{\Gamma(\alpha+\beta+1-s)} \mathfrak{M}\{f(x) ;, \\
& \mathscr{N}[\mathcal{R}\{\alpha, \beta: f(x)\}]=\frac{\Gamma(\beta+s)}{\Gamma(\alpha+\beta+s)} \mathfrak{M}\{\{f(x)\} .
\end{aligned}
$$

For a discussion of these transforms see Erdelyi [1] and Kober [3]. 
THEOREM 2. If (i) $\alpha>0, \beta>0$, (ii) $f(x) \in L(0, \infty)$, (iii) $x^{-1 / 2} f(x) \in$ $L(0, \infty)$, where $f(y)$ is of bounded variation near the point $y=x$, (iv) $\mathfrak{M}\{f(x)\}=F(s) \in L\left(\frac{1}{2}-i \infty, \frac{1}{2}+i \infty\right)$ and (v) $x^{-1 / 2} \mathfrak{I}\{\alpha, \beta: f(x)\} \in L(0, \infty)$ and is of bounded variation near $y=x$ then

$$
x^{-\alpha-\beta} \mathfrak{Q}^{-1}\left[t^{-\alpha} \mathfrak{L}\left\{x^{\beta} f(x)\right]=\mathfrak{J}\{\alpha, \beta: f(x)\} .\right.
$$

Proof. From conditions (i) and (ii) and Theorems 2 and 5(a) of [3] both $\mathfrak{I}\{\alpha, \beta: f(x)\}$ and $\Re\{\alpha, \beta: f(x)\}$ exists and belong to $L(0, \infty)$ and also (30) and (31) hold. From (iii) and Theorem 28, p. 46 of [7], with $k=\frac{1}{2}$, we have

$$
f(x)=\frac{1}{2 \pi i} \int_{1 / 2-i \infty}^{1 / 2+i \infty} F(s) x^{-s} d s .
$$

It is necessary to work with $x^{s}$ instead of $x^{-s}$ so in (33) we replace $s$ by $1-s$ first and then apply the operator $\mathfrak{L} x^{\beta}$. The result is

$$
\mathfrak{L}\left\{x^{\beta} f(x)\right\}=\int_{0}^{\infty} e^{-t x} x^{\beta}\left\{\frac{1}{2 \pi i} \int_{1 / 2-i \infty}^{1 / 2+i \infty} F(1-s) x^{s-1} d s\right\} d x .
$$

Along the line $s=\frac{1}{2}+i \tau$, the real part of the power of $x$ is $\beta-\frac{1}{2}$. From (iv) we easily deduce that $F(1-s) \in L\left(\frac{1}{2}-i \infty, \frac{1}{2}+i \infty\right)$ and, from (i), $\beta-\frac{1}{2}>-\frac{1}{2}$ and therefore the double integral in (34) is absolutely convergent. We may then integrate first with respect to $x$, giving us

$$
\mathfrak{L}\left\{x^{\beta} f(x)\right\}=\frac{1}{2 \pi i} \int_{1 / 2-i \infty}^{1 / 2+i \infty} \Gamma(\beta+s) t^{-\beta-s} F(1-s) d s .
$$

From (35) we have

$$
\begin{aligned}
& \mathfrak{L}^{-1}\left[t^{-\alpha} \mathfrak{L}\left\{x^{\beta} f(x)\right\}\right] \\
& (36)=\mathfrak{L}^{-1}\left[\frac{1}{2 \pi i} \int_{1 / 2-i \infty}^{1 / 2 ; i x} \Gamma(\alpha+\beta+s) \frac{\Gamma(\beta+s)}{\Gamma(\alpha+\beta+s)} t^{-\alpha-\beta-s} F(1-s) d s\right] .
\end{aligned}
$$

Now (iv) also implies that $F(1-s) \in L\left(\frac{1}{2}-i \infty, \frac{1}{2}+i \infty\right)$. Hence from the asymptotic expansion of the gamma function [10, p. 273] we have

$$
\Gamma(\beta+s) F(1-s) / \Gamma(\alpha+\beta+s)=O\left\{s^{-\alpha} F(1-s)\right\} .
$$

Hence, since $x>0$ by condition (i), from (iv) we have

$$
\Gamma(\beta+s) F(1-s) / \Gamma(\alpha+\beta+s) \in L\left(\frac{1}{2}-i \infty, \frac{1}{2}+i \infty\right) .
$$

(38) justifies us in using equation (4) of [2] with $\alpha=1$ and $\beta$ replaced by $\alpha+\beta$. The result is that the $\mathfrak{L}^{-1}$ operator in (36) eliminates $\Gamma(\alpha+\beta+s)$ 
from the numerator of the integrand in (36). This gives us

$$
\mathfrak{L}^{-1}\left[t^{-\alpha} \mathfrak{L}\left\{x^{\beta} f(x)\right\}\right]=\frac{1}{2 \pi i} \int_{1 / 2-i \infty}^{1 / 2+i \infty} \frac{\Gamma(\beta+s)}{\Gamma(\alpha+\beta+s)} x^{\alpha+\beta+s-1} F(1-s) d s .
$$

On replacing $s$ in (39) by $1-s$ and using (30) we have

$$
\mathfrak{L}^{-1}\left[t^{-\alpha} \mathfrak{L}\left\{x^{\beta} f(x)\right\}\right]=\frac{1}{2 \pi i} x^{\alpha+\beta} \int_{1 / 2-i \infty}^{1 / 2+i \infty} \mathfrak{M}[\mathfrak{I}\{\alpha, \beta: f(x)\}] x^{-s} d s .
$$

Finally from (v) and Theorem 29, p. 46 of [7], with $k=\frac{1}{2}$, we deduce that

$$
x^{-\alpha-\beta} \mathfrak{L}^{-1}\left[t^{-\alpha} \mathfrak{L}\left\{x^{\beta} f(x)\right\}\right]=\mathfrak{I}\{\alpha, \beta: f(x)\} .
$$

This completes the proof. To test Theorem 2, let $f(x)=e^{-1 / x}$. From [5, p. 146, (29)] with $\nu=1+\beta$ and $a=4$ we have

$$
\mathfrak{L}\left\{x^{\beta} e^{-1 / x}\right\}=2 t^{-(1 / 2)(1+\beta)} K_{1+\beta}\left(2 t^{1 / 2}\right) .
$$

From [5, p. 283, (43)], with $a=1, \mu-\frac{1}{2}=-\alpha-\frac{1}{2} \beta$ and $2 \nu=1+\beta$ we have

$$
\mathfrak{I}\left\{\alpha, \beta: e^{-1 / x}\right\}=x^{-\beta / 2} e^{-1 / 2 x} W_{-\alpha-\beta / 2,(1 / 2)(1+\beta)}(1 / x),
$$

where $K_{v}(x)$ is a Bessel function of the second kind $\left[9\right.$, p. 78] and $W_{k, \mu}(x)$ is Whittaker's function of (1) and (2). There appear to be no tables for $\mathfrak{I}\{\alpha, \beta: f(x)\}$ but there are some for $x^{\alpha} \mathfrak{I}\{\alpha, 0: f(x)\}$ in [6].

From (43) we have

$$
x^{\alpha} \mathfrak{I}\left\{\alpha, 0: e^{-1 / x}\right\}=x^{\alpha} e^{-1 / 2 x} W_{-x, 1 / 2}\left(x^{-1}\right),
$$

which checks with [6, p. 187, (18)], with $\nu=1, \mu=\alpha$ and $a=1$.

THEOREM 3. With the same conditions as in Theorem 2, except that $\mathfrak{I}\{\alpha, \beta: f(x)\}$ is replaced by $\mathfrak{R}\{\alpha, \beta: f(x)\}$, we have

$$
\left\{x^{1-\alpha-\beta} \mathfrak{Q}^{-1}\left[t^{-\alpha} \mathfrak{Q}\left\{x^{\beta-1} f(1 / x)\right\}\right]\right\}_{x=1 / X}=\Re\{\alpha, \beta: f(X)\} .
$$

The proof closely follows that of Theorem 2. Briefly we start with (33) and replace $x$ by $x^{-1}$. The operators $\mathcal{L}$ and $\mathfrak{L}^{-1}$ introduce $\Gamma(\beta+s) / \Gamma(\alpha+\beta+s)$ into the integrand, as in (39). From (32) the integrand then contains $\mathfrak{M}[\mathcal{R}\{\alpha, \beta: f(x)\}] x^{\alpha+\beta+s-1}$. On replacing $x$ by $1 / X$ and using (33) again, (45) is established.

To test (45) we let $f(x)=e^{-x}$. From [5, p. 146, (29)] we have

$$
\mathfrak{L}\left\{x^{\beta-1} e^{-1 / x}\right\}=2 t^{-\beta}{ }^{2} K_{\beta}\left(2 t^{1 / 2}\right) .
$$


From [5, p. 283, (43)], with $a=1, \mu-\frac{1}{2}=\frac{1}{2}-\alpha-\frac{1}{2} \beta$ and $2 v=\beta$ we have finally

$$
\mathcal{R}\left\{\alpha, \beta: e^{-x}\right\}=X^{\beta / 2-1 / 2} e^{-X / 2} W_{1 / 2-\alpha-\beta / 2, \beta / 2}(X),
$$

where the $K$ and $W$ functions are as in the test for Theorem 2 .

There appear to be no tables for $\mathcal{R}\{\alpha, \beta: f(X)\}$ but in [6] there are tables for $X^{\alpha} \Re\{\alpha,-\alpha: f(X)\}$. Since $W_{1 / 2-\alpha / 2,-\alpha / 2}(X)=X^{(1-\alpha) / 2} e^{-X^{\prime} / 2}[6$, p. 432] from (47) we have

$$
X^{\alpha} \Re\left\{\alpha,-\alpha: e^{-X}\right\}=e^{-x}
$$

which agrees with $[6$, p. 202, (11)] when $a=1$. This serves as a check for (45).

\section{REFERENCES}

1. A. Erdélyi, On some functional transfornations, Univ. e Politecnico Torino Rend. Sem. Mat. 10 (1951), 217-234. MR 13, 937.

2. C. Fox, Solving integral equations by $L$ and $L^{-1}$ operators, Proc. Amer. Math. Soc. 29 (1971), 299-306.

3. H. Kober, On fractional integrals and derivatives, Quart. J. Math. Oxford Ser. 11 (1940), 193-211. MR 2, 191.

4. R. K. Saxena, An inversion formula for the Varma transform, Proc. Cambridge Philos. Soc. 62 (1966), 467-471. MR 33 \#4613.

5. A. Erdélyi et al. (Editors), Tables of integral transforms. Vol. 1, McGraw-Hill, New York, 1954.

6. - Tables of integral transforms. Vol. 2, McGraw-Hill, New York, 1954.

7. E. C. Titchmarsh, Introduction to the theory of Fourier integrals, Clarendon Press, Oxford, 1937.

8. R. S. Varma, On a generalization of the Laplace integral, Proc. Nat. Acad. Sci. India Sect. A 20 (1951), 209-216. MR 15, 868.

9. G. N. Watson, A treatise on the theory of Bessel functions, Cambridge Univ. Press, Cambridge; Macmillan, New York, 1922.

10. E. T. Whittaker and G. N. Watson, A course of modern analysis, Cambridge Univ. Press, New York, 1915.

Department of Mathematics, Sir George Williams University, Montreal, 107, QUebec, CANADA 